\title{
Characterization of Elastic Properties of Hard Carbon and Boron Nitride Films using the Brillouin Light Scattering Technique
}

\author{
T. Wittkowski, V. Wiehn, J. Jorzick, K. Jung, and B. Hillebrands \\ Fachbereich Physik und Schwerpunkt Materialwissenschaften, Universität Kaiserslautern, Erwin-Schrödinger-Str. 56, 67663 Kaiserslautern, \\ Germany
}

\begin{abstract}
The elastic properties of thin graphitic carbon and hexagonal boron nitride films have been determined by Brillouin light scattering from thermally excited surface acoustic phonons. The carbon films have been grown by plasma beam deposition at $420{ }^{\circ} \mathrm{C}$. The $\mathrm{BN}$ films have been deposited by rf-magnetron sputtering and simultaneous ion plating. In the case of amorphous carbon a layered substructure has been found with a rather compliant intermediate layer at the interface. The stiffness of the films increases with increasing ion energy. As for carbon the BN coatings exhibit a pronounced elastic anisotropy indicating a preferred orientation of the hexagonal planes perpendicularly to the surface. The comparison of the Young's moduli determined by load indentation and by Brillouin light scattering allows the conclusion that the material is composed of two or more components of different stiffness.
\end{abstract}

Films grown by magnetron sputtering or plasma beam deposition are in general nanocrystalline or amorphous and do not exhibit single crystal properties. Their elastic properties need to be determined experimentally because they depend strongly on the deposition conditions, and they can in general not be derived from theoretical considerations. Brillouin light scattering (BLS) is a well established technique to obtain information on elastic properties of thin films by measuring the frequency shift of light scattered inelastically from phonons $[1,2]$. The information obtained by BLS usually allows to determine several independent elements of the elastic tensor and to reveal the existence of an interlayer between film and substrate.

\section{Sample Preparation}

Boron nitride films have been grown on (100)-silicon by 13.56 $\mathrm{MHz}$ rf magnetron sputtering from a polycrystalline stoichiometric BN target in a pure argon atmosphere of about $4 \cdot 10^{-4}$ mbar. The apparatus has been described in detail earlier [3]. On account of the unbalanced magnet configuration a rather high flux of argon ions can be extracted from

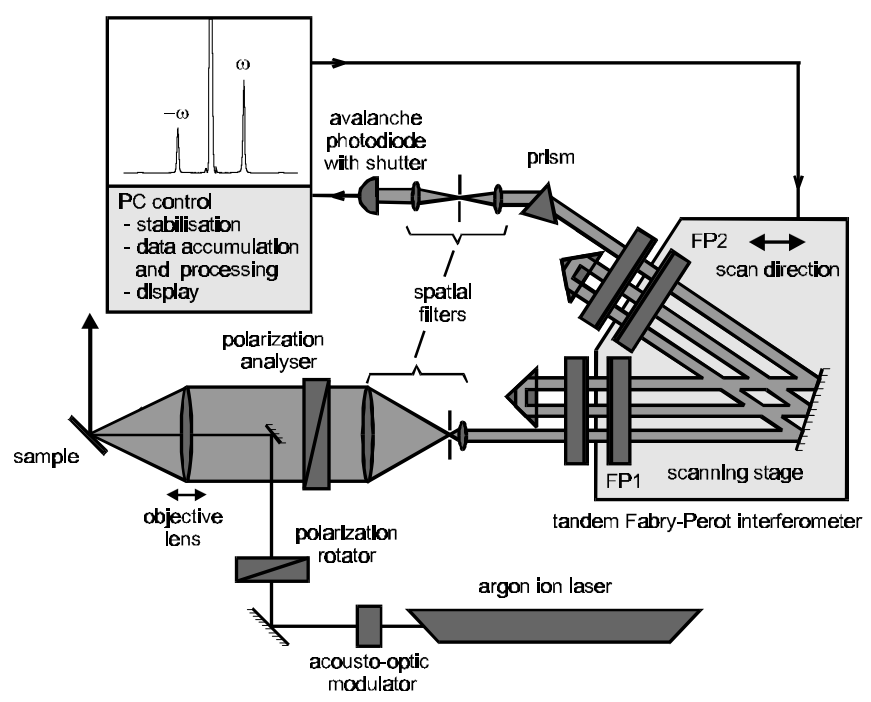

Fig. 1: Brillouin light scattering spectroscopy setup in backscattering geometry. the plasma by applying a rf bias to the substrate. The energy of the ions was near $130 \mathrm{eV}$. At an ion current density of 1.2 $\mathrm{mA} / \mathrm{cm}^{2}$ at the substrate the growth rate was found to be 6.2 $\mathrm{nm} / \mathrm{min}$. The argon ion flux to the substrate was about a factor of 7 larger than the flux of boron and nitrogen atoms contributing to the film growth. The substrate temperature was held constant at $350{ }^{\circ} \mathrm{C}$. Under these conditions boron nitride films grow in the hexagonal modification [4,5] which has been controlled by infrared spectroscopy.

Carbon films with a hydrogen content of 5\% have been produced by plasma beam deposition on a (001) silicon substrate heated to $420{ }^{\circ} \mathrm{C}$. The plasma beam source, which has been described in detail previously in [6], consists of a capacitive, magnetically confined r.f. discharge. Acetylene was used as working gas because it forms almost exclusively $\mathrm{C}_{2} \mathrm{H}_{2}{ }^{+}$-ions in low pressure plasmas [6]. The fraction of this ion species was larger than $95 \%$ at a background pressure of $6 \cdot 10^{-5}$ mbar. The films presented here were grown at ion energies corresponding to kinetic energies of 83 and $120 \mathrm{eV}$ per carbon atom [7].

\section{Brillouin Light Scattering}

In Brillouin light scattering (BLS) photons are inelastically scattered from acoustic phonons. Figure 1 shows the BLS spectrometer [8]. The $5145 \AA$ line of a single mode $\mathrm{Ar}^{+}$-laser is focused onto the sample. The incoming and the scattered light is polarized in the sagittal plane which is defined by the wave vector of the incident light and the surface normal ( $p-p$ polarization). The experiment is performed in backscattering geometry for maximum momentum transfer. In the case of an ideally flat surface no elastic scattering contribution would enter the spectrometer. In a real experiment the elastically scattered light causes even in this configuration the most intense peak in the spectrum. The scattered light is analyzed in a $(3+3)$-tandem Fabry-Perot-interferometer with a contrast better than $1: 10^{10}[8,9]$. The tandem configuration removes the ambiguity of the periodical transmission function of a single interferometer. The spectral resolution of the interferometer is about $1 \%$ of the free spectral range which was chosen to be $38 \mathrm{GHz}$. An acousto-optical modulator prevents the detector (avalanche photodiode) from 
overload in the transmission maxima of the elastically scattered laser light.

Figure 2 schematically presents the scattering geometry. The wavevectors of the incident and the scattered light are $\vec{k}_{0}$ and $\vec{k}_{s}$, respectively. Taking into account $\vec{k}_{0} \approx-\vec{k}_{s}$, momentum conservation of the components parallel to the surface requires $\left|\vec{q}_{\|}\right|=2\left|\vec{k}_{0}\right| \cdot \sin \theta=4 \pi / \lambda_{\text {Laser }} \cdot \sin \theta$, where $\mathrm{q}_{\|}$is the phonon wavevector parallel to the surface and $\theta$ is the angle between the surface normal and the photon wave vector. Because of energy conservation the phonon frequency is given by the frequency shift $\Delta v$ of the scattered light. Whereas the wavevectors of the photons are comparable to those of the phonons their energies are several magnitudes larger than those of the phonons. In general several surface waves are detected. By varying $\theta$ the dispersion of the phase velocity $\mathrm{v}\left(\mathrm{q}_{\|}\right)=2 \pi \cdot \Delta v / \mathrm{q}_{\|}=\Delta v \cdot \lambda_{\text {Laser }} /(2 \sin \theta)$ of these surface waves can be determined.

Considering a semi-infinite bulk sample, the only existing mode localized at the surface of the half space and polarized parallel to the surface normal is called the Rayleigh mode [10]. In the case of a thin film supported by a substrate additional modes will appear. These modes are localized at the boundary between film and substrate (Stoneley modes) or they are inner film modes due to reflections at the boundaries (Sezawa modes). Beyond these discrete modes there will exist a continuous spectrum as well as resonances and mixed modes due to the semi-infinite substrate; details are discussed in [12]. A theoretical description of the light scattering process includes two scattering mechanisms, namely the modulation of the dielectric tensor (elasto-optical effect) and the dynamical corrugation of the surface (ripple effect) by thermal vibrations [11]. For the discrete as well as for the continuous modes both the ripple effect and the elastooptical modulation determine the cross section of the light scattering process. A detailed description of the theory of the Brillouin light scattering for $\mathrm{p}$ and s polarization of the incident and the scattered light is given in a review article by Nizzoli et al. [12].

The wavelength of surface acoustic phonons under investigation in this study is much larger than the lattice constant so

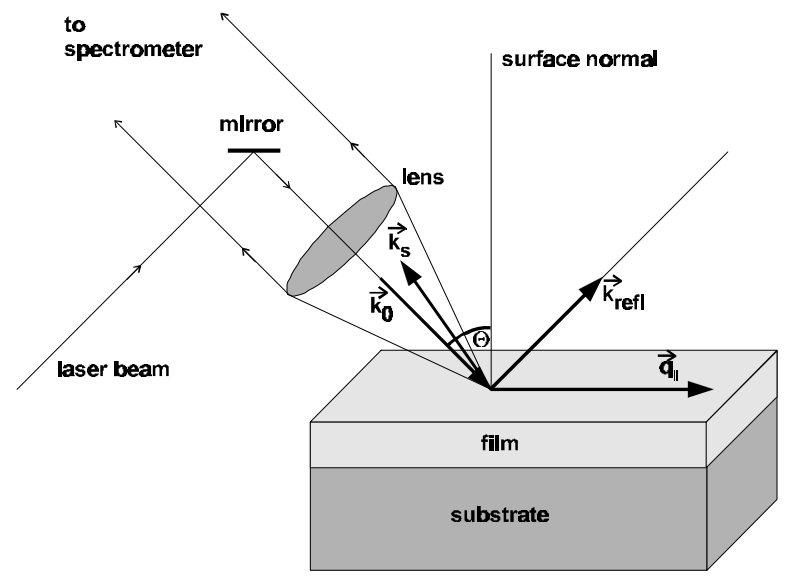

Fig. 2: Schematic diagram of the scattering geometry with the wavevectors of the incident and inelastically scattered light, $\vec{k}_{0}$ and $\vec{k}_{s}$. From $\vec{k}_{0} \cong-\vec{k}_{s}$ follows $\left|\vec{q}_{\|}\right|=2\left|\vec{k}_{0}\right| \cdot \sin \Theta$ where $\vec{q}_{\|}$is the phonon wavevector parallel to the surface. $\vec{k}_{r e f l}$ indicates the wavevector of the elastically scattered light. that accessible wavevectors lie in a range of about $0.1 \%$ of the Brillouin zone around the zone center. This allows the description of acoustic modes in a continuum mechanical model without the need to take the interatomic forces into account explicitly. To extract the elastic constants from the phonon phase velocities the underlying continuum mechanical model implies the integration of the equation of motion [13]. Suitable linear combinations of the obtained partial waves must obey boundary conditions. These conditions are the continuity of the displacement field and the continuity of the stress normal to the film at the interfaces. Amplitudes of waves propagating into the substrate must decrease. For homogenous films it is found that the sound velocities of the localized modes only depend on the dimensionless product of $\mathrm{q}_{\|}$and the film thickness $\mathrm{h}$ [13].

In the experiment $\mathrm{q}_{\|}$depends on the angle of incidence $\theta$ of light which is varied from $\sin \theta=0.3$ to $\sin \theta=0.95$. This range is rather narrow, thus it is necessary to examine films of different thicknesses $h$ to access a wide $q \|$ h-range of the dispersion curves. If the films are deposited under identical conditions and if their elastic properties do not depend on the thickness the combined phonon dispersion curves of the surface wave modes are continuous and will correlate with one single set of elastic constants. If, on the other hand, the dispersion curves do not fit to each other in overlapping regions the films must have a layered substructure.

Films deposited with medium or high ion fluxes, as in this study, normally build up an internal stress. The material at the interface not yet exposed to the full stress is often less compact than the material on top. In the ongrowing film the material can even undergo phase transitions due to the increasing stress. Because of the different penetration depth a thin layered substructure at the interface will affect the phase velocity of the Sezawa modes more than that of the Rayleigh mode. The influence of a thin interlayer on the phase velocities will decrease with increasing film thickness.

\section{Results and Discussion}

For the h-BN films the dispersion of surface waves was investigated in a $\mathrm{q} \|$ h range between 0.2 and 14 by using films of different thicknesses deposited under identical coating conditions as well as by varying the angle of incidence from $17.5^{\circ}$ to $72^{\circ}$. The combined results of these measurements are shown in Figure 3. The horizontal error bars are mainly due to uncertainties in the determination of the film thickness. The error in sound velocity is due to inaccuracies in the determination of the peak positions in the Brillouin spectra. Except for the slight discontinuity between the data of the two thinnest films the dispersion curves of different samples merge quite well. Therefore it can be assumed that the films do not exhibit a pronounced layered substructure.

The mode with the lowest velocity is the Rayleigh surface mode. Its velocity, $\mathrm{v}_{\mathrm{R}}$, is constant for the thickest film, i.e. the Rayleigh mode is no more influenced by the substrate and it reflects the elastic properties of the film material only. If the film thickness approaches zero, the velocity of the Rayleigh mode will be equal to the velocity of the Rayleigh mode of the silicon substrate which is found to be $5074 \mathrm{~m} / \mathrm{s}$. For phase velocities above the threshold velocity of the sub- 
strate, $\mathrm{v}_{\mathrm{T}}^{\mathrm{S}}$, which is the lowest bulk velocity for the given direction of propagation and polarization, the continuation of the Sezawa mode into this regime of substrate bulk modes will show up as a resonance. Only the discrete spectrum is used for the evaluation of the elastic constants by comparing the experimental with the corresponding calculated sound velocities.

Figure 4 shows on an enlarged vertical scale a subsection of the previous figure with sound velocities below $\mathrm{v}_{\mathrm{T}}^{\mathrm{s}}$. The calculated dispersion curves of the discrete spectrum are obtained in a least squares fit to the experimental data indicated by squares and triangles, using the elastic constants as free parameters. The data points indicated by circles are not fitted because the assignment to the correct mode order is ambiguous. The best fit result, which is characterized by a minimum in the $\chi^{2}$ sum, clearly demonstrates that the experimental data points, indicated by triangles in Figure 5, have to be assigned to the $2^{\text {nd }}$ Sezawa mode. Other assignments lead to unsatisfactory fit results. Probably the $1^{\text {st }}$ Sezawa mode is not visible because of a too small scattering cross section. Possible reasons for such a low cross section could be a phonon polarization of mainly longitudinal character for the mode to be observed and/or a small total scattering amplitude due to cancelling ripple and the elastooptical contributions as it is known, e.g. for GaAs [14]. Because the elasto-optical coupling of the material is not known the effect of the latter contribution is difficult to estimate.

The stiffness constants which correspond to the best fit are $c_{11}=65 \mathrm{GPa}, \mathrm{c}_{13}=7 \mathrm{GPa}, \mathrm{c}_{33}=92 \mathrm{GPa}$ and $\mathrm{c}_{44}=53 \mathrm{GPa}$ with standard deviations of $3 \%, 35 \%, 14 \%$ and $6 \%$, respectively. Although the bonding between the hexagonal planes is weak the sputtered material is rather stiff. This is comparable to the observations in the carbon system where amorphous carbon films with mainly $\mathrm{sp}^{2}$-bonded atoms are called diamondlike because the strong $\mathrm{sp}^{2}$ bondings form a stable three-dimensional network.

The elastic anisotropy, defined as the ratio of the stiffnesses parallel and perpendicular to the film surface, $c_{11} / c_{33}$, amounts to 0.7. McKenzie et al. [15] and Cardinale et al. [16] discuss the preferred crystallite orientation of h-BN

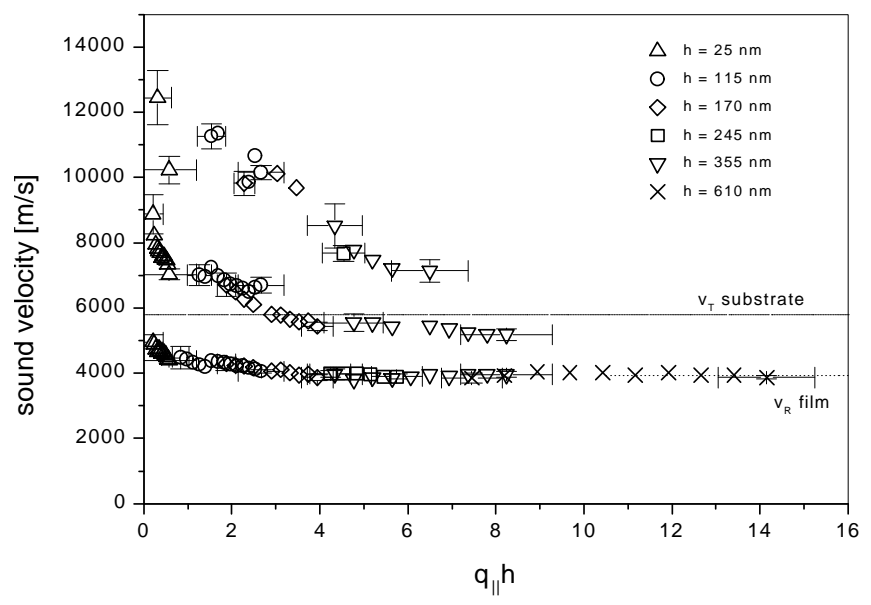

Fig. 3: Dispersion curves of six samples with thicknesses h from 25 to 610 $\mathrm{nm}$. The Rayleigh mode is observed in the spectra of each sample. The dotted lines indicate the threshold velocity, $v_{T}$, of the substrate in [011]direction and the velocity of the Rayleigh mode, $v_{R}$, for a thick film. under a compressive biaxial stress. This internal stress is induced during the growing process and depends on the energy and momentum transfer of the impinging ions as well as on the substrate temperature. The elastic anisotropy of 0.7 determined in the present study indicates the h-BN nanocrystallites to be non-arbitrarily oriented. A comparison of $\mathrm{c}_{11} / \mathrm{c}_{33}$ with the single crystal stiffness constants leads to the conclusion that the c-axes of the crystallites have a statistically preferred orientation parallel to the surface.

To characterize the elastic properties of the film material with an independent method and also to determine its hardness, ultralow load indentation measurements with a "Nanotest 300" Berkovitch indenter have been performed. The hardness is found to be $14.6 \pm 0.3 \mathrm{GPa}$ for the $\mathrm{h}$-BN films and $12.0 \pm 0.4 \mathrm{GPa}$ for the silicon substrate with (100) surface. The Young's moduli are $\mathrm{E}_{\mathrm{BN}} /\left(1-\mathrm{v}_{\mathrm{BN}}{ }^{2}\right)=177 \pm 3$ $\mathrm{GPa}$ and $\mathrm{E}_{\mathrm{Si}} /\left(1-v_{\mathrm{Si}}{ }^{2}\right)=152 \pm 2 \mathrm{GPa}$ for the film and the substrate, respectively. These results, indicating the film to possess a higher stiffness than the substrate, are rather surprising because the elastic constants of the BN film found by BLS were smaller than the corresponding constants of the silicon substrate.

The discrepancy has probably systematic reasons, which are due to the specific morphology of the material. It is assumed that the material consists of two or more components with distinctly different elastic properties. It is well known from many investigations that $\mathrm{BN}$ films deposited by various methods form a complex morphology with crystallites embedded in an amorphous matrix [17-20]. Especially if the material is subjected to an intense ion bombardment during the growing process it tends to form crystallites on the nanometer scale. The inhomogeneity must be on a length scale which is small compared to the wavelength of the surface phonons. Otherwise no clear peaks would be observed in the Brillouin light scattering spectra. Such a partly nanocrystalline material will contain several constituents with strongly different elastic constants. Besides the amorphous component, which is essentially isotropic, the h-BN crystal-

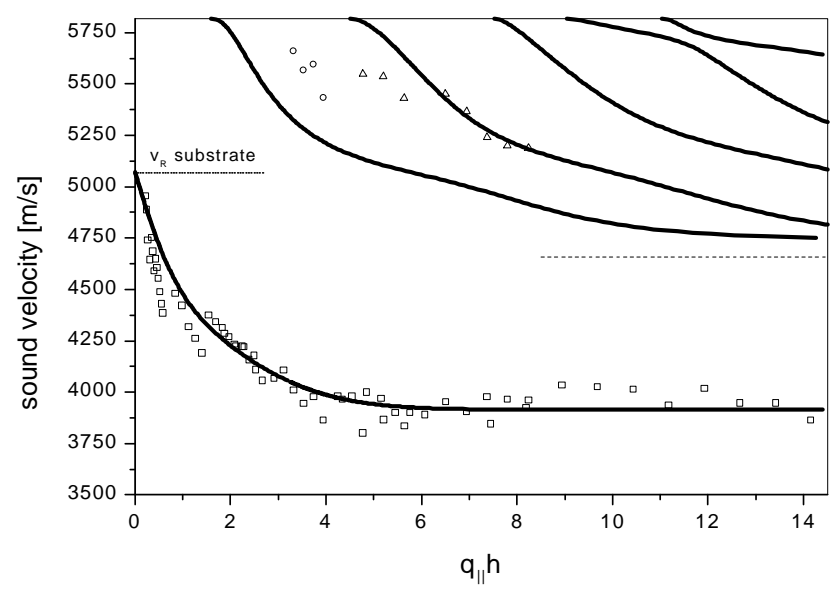

Fig. 4: Dispersion curves of the Rayleigh mode (squares) and the $2^{\text {nd }}$ Sezawa mode of the $355 \mathrm{~nm}$ thick sample (triangles). The lines indicate the best fit to the experimental data. The corresponding parameters are the stiffness constants $\mathrm{c}_{11}=65 \mathrm{GPa}, \mathrm{c}_{13}=7 \mathrm{GPa}, \mathrm{c}_{33}=92 \mathrm{GPa}, \mathrm{c}_{44}=53 \mathrm{GPa}$ and the mass density $\tilde{\mathrm{n}}=2.14 \mathrm{~g} / \mathrm{cm}^{3}$. The dotted line represents the velocity of the substrate Rayleigh mode. The dashed line indicates the lowest velocity parallel to the surface of the bulk modes in the film material. Except for the Rayleigh wave all dispersion curves asymptotically approach this velocity. 


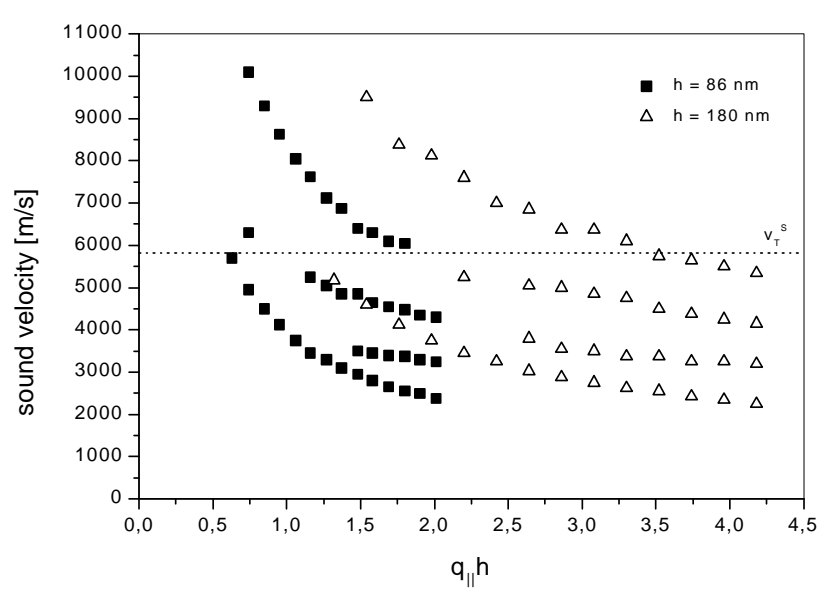

Fig. 5: Combined dispersion curves of two samples of different thickness $h$ grown with ion energies of $120 \mathrm{eV} / \mathrm{C}$-atom at $420{ }^{\circ} \mathrm{C}$. The waves propagate in [110]-direction of the (001) silicon substrate. The dotted line indicates the velocity threshold. For both films the Rayleigh mode and three Sezawa modes are detected.

lites will behave differently if the stress is exerted parallel or perpendicular to the c-axis of the hexagonal lattice. Such a film would behave differently in BLS and load indentation measurements because the extend of elongation of the material is very different. In the case of the Brillouin light scattering the displacement of the atoms is only a small fraction of the internuclear distance. The measurement will be mainly sensitive to the more compliant component. In the case of the load indentation the material is elongated far beyond the limit of plastic deformation. The elastic response of the deformed material is measured in the unloading process immediately following the indentation. Such a method will preferentially detect the stiff component of the material.

In figure 5 experimentally found dispersion curves of two carbon films with thicknesses of 86 and $180 \mathrm{~nm}$, deposited under identical conditions with ion energies of $120 \mathrm{eV}$ are depicted. The dotted line which indicates the threshold velocity of the substrate separates the discrete part from the continuous part of the spectrum. The dispersion curves of the two samples do not coincide. The thicker film with the larger sound velocities for given $\mathrm{q}_{\|} \mathrm{h}$-values obviously possesses a much higher overall stiffness than the thinner film. This effect can only be explained by assuming that the films exhibit a pronounced layered substructure with a more compliant material at the substrate interface. Despite of this fact least squares fits to the experimental data have been performed under the assumption that the films are homogeneous to get some sort of averaged elastic constants. The results for four samples are summarised in table 1. In the energy interval studied here the stiffness increases with increasing energy per carbon atom. The stiffening with increasing thick-
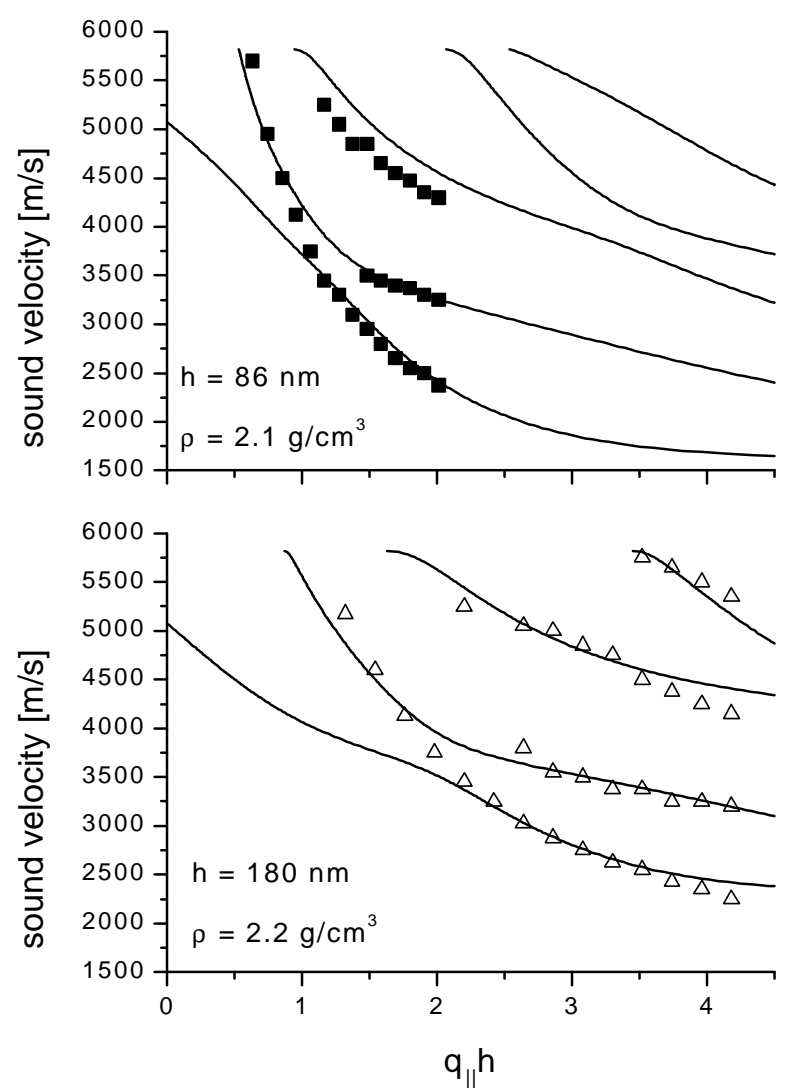

Fig. 6: Dispersion curves of the films as presented in figure 5. Measured data (points) and fit curves (lines) of the discrete part of the spectrum are shown on an enlarged scale for the thin and the thicker film in the upper and lower plot, respectively. The fit is performed under the assumption that each film is homogeneous, the results are presented in table 1.

ness is most pronounced for the samples deposited at 120 $\mathrm{eV} / \mathrm{C}$-atom.

This variation in elastic behaviour cannot be described by a stress induced phase transition from $\mathrm{sp}^{2}$ to $\mathrm{sp}^{3}$ bondings which is observed for deposition of amorphous carbon at room temperature. It is well described by the subplantation process [21-23]. As Sattel et al. could show [23], heating the substrate to temperatures over about $250{ }^{\circ} \mathrm{C}$ prevents the film material to become diamond-like. On the other hand films grown at temperatures above $400^{\circ} \mathrm{C}$ become highly efficient nucleation sources for diamond growth. Using these films as substrates in a microwave-CVD system the nucleation density is enlarged dramatically from $10^{4}$ to $10^{8} \mathrm{~cm}^{-2}$ at the temperature threshold [24]. Since diamond does not grow well on amorphous $\mathrm{sp}^{3}$ bonded carbon, the nucleation centers are characterized as to probably consist of ordered graphitic carbon [24]. This phase transition from amorphous to crystalline graphitic carbon coincides with a steep decrease in the H-content to about 5\% [23]. Due to dehydroge-

\begin{tabular}{|l|l|l|l|l|l|}
\hline $\begin{array}{l}\text { ion energy [eV/C- } \\
\text { atom] }\end{array}$ & film thickness $[\mathrm{nm}]$ & $\begin{array}{l}\mathrm{c}_{11} \\
{[\mathrm{GPa}]}\end{array}$ & $\begin{array}{l}\mathrm{c}_{13} \\
{[\mathrm{GPa}]}\end{array}$ & $\begin{array}{l}\mathrm{c}_{33} \\
{[\mathrm{GPa}]}\end{array}$ & $\begin{array}{l}\mathrm{c}_{44} \\
{[\mathrm{GPa}]}\end{array}$ \\
\hline 80 & 83 & $32 \pm 6$ & $28 \pm 6$ & $35 \pm 8$ & $4 \pm 0.9$ \\
\hline 80 & 145 & $52 \pm 4$ & $50 \pm 6$ & $71 \pm 9$ & $4 \pm 0.4$ \\
\hline 120 & 86 & $37 \pm 7$ & $27 \pm 6$ & $37 \pm 9$ & $7 \pm 1.1$ \\
\hline 120 & 180 & $91 \pm 21$ & $117 \pm 35$ & $195 \pm 60$ & $22 \pm 2$ \\
\hline
\end{tabular}

Table 1: Elastic constants of four amorphous carbon films obtained from least squares fits to the measured phonon dispersion curves under the assumption of homogeneous films. 
nation the dangling carbon bonds cause an increase in reactivity of the residual surface atoms. Additional energy input by the impinging particles may enhance the formation of $\mathrm{sp}^{2}$ bonded crystallites in the investigated energy range from 83 to $120 \mathrm{eV} / \mathrm{C}$-atom. Thus the increased stiffness of the thicker films is attributed to the rapid change in morphology. Thick films exhibit a distinct elastic anisotropy whereas thin films seem to be essentially isotropic with $1 / 2\left(c_{11}-c_{12}\right)=c_{44}$ and $c_{11} / c_{33} \approx 1$. Analogously to the results on h-BN presented here, the c-axes of the hexagonal crystallites are preferentially oriented parallel to the surface. This effect which has already been described above for the boron nitride system is a consequence of biaxial compressive stress induced during deposition, and it is a necessary condition to accomodate the mismatch in lattice parameters for diamond growth $[15,25]$.

\section{Conclusion}

In conclusion it was shown on the basis of graphitic carbon and polycrystalline boron nitride films that Brillouin light scattering is an excellent tool to characterize the elastic behaviour of hard films and to gain deeper insight into the morphology of coating materials. Financial support by the Deutsche Forschungsgemeinschaft is gratefully acknowledged.

\section{References}

[1] G.I. Stegemann, J.A. Bell, W.R. Duda, C.M. Falco, U.J. Gibson, B. Hillebrands, W. Knoll, L.A. Laxhuber, Suk Mok Lee, J. Makous, F. Nizzoli, C.T. Seaton, J.D. Swalen, G. Wegner, R. Zanoni, Scattering in Volumes and Surfaces, M. Nieto-Vesperinas, J.C. Dainty (Editors), Elsevier (1990).

[2] B. Hillebrands, P. Krams, K. Spörl, D. Weller, J. Appl. Phys. 69 (1991) 938.

[3] T. Wittkowski, J. Jorzick, K. Jung and B. Hillebrands, submitted to Thin Solid Films.

[4] C.A. Davis, Thin Solid Films 226 (1993) 30

[5] S. Ulrich, J. Scherer, J. Schwan, I. Barzen, K. Jung, H. Erhardt, Diam. Rel. Mat. 4 (1995) 288.

[6] M. Weiler, J. Robertson, S. Sattel, V.S. Veerasamy, K. Jung, H. Ehrhardt, Diam. Rel. Mat. 4 (1995), 268.

[7] M. Weiler, S. Sattel, T. Giessen, K. Jung, H. Ehrhardt, V.S. Veerasamy, J. Robertson, Phys. Rev. B 53 (1996) 1594.

[8] R. Mock, B. Hillebrands, J.R. Sandercock, J. Phys. E 20 (1987) 657.

[9] B. Hillebrands, Rev. Sci. Instrum. 70 (1999) 1589.

[10]Lord Rayleigh, Proc. London Math. Soc. 17 (1887) 4.
[11]A.M. Marvin, V. Bortolani, F. Nizzoli, J. Phys. C: Solid State Phys. 13 (1980) 299.

[12] V. Bortolani, A.M. Marvin, F. Nizzoli, G. Santoro, J. Phys. C: Solid State Phys. 16 (1983) 1757.

[13] G.W. Farnell, E.L. Adler, Physical Acoustics edited by W.P. Mason, R.N. Thurston (Academic Press, N.Y., 1972) 9, 35

[14] G. Carlotti, D. Fioretto, L. Giovannini, F. Nizzoli, G. Socino, L. Verdini, J. Phys.: Condens. Matter 4 (1992) 257.

[15]D.R. McKenzie, W.D. McFall, S.Reisch, B.W. James, I.S. Falconer, R.W. Boswell, H. Persing, A.J. Perry, A. Durandet, Surface And Coatings Techn. 78 (1996) 255.

[16] G.F. Cardinale, D.L. Medlin, P.B. Mirkarimi, K.F. McCarty, D.G. Howitt, J. Vac. Sci. Technol. A 15 (1997) 196.

[17] W. Donner, S. Chamera, A. Rühm, H. Dosch, S. Ulrich, H. Ehrhardt, Appl. Phys. A 65 (1997) 1.

[18]D.R. McKenzie, W.D. McFall, W.G. Sainty, C.A. Davis, R.E. Collins, Diam. Rel. Mat. 2 (1993) 970.

[19] W. Dworschak, K. Jung, H. Ehrhardt, Thin Solid Films 254 (1995) 65.

[20]T. Klotzbücher, W. Pfleging, D.A. Wesner, M. Mergens, E.W. Kreutz, Diam. Rel. Mat. 5 (1996) 525.

[21] J. Robertson, Diam. Rel. Mat. 3 (1994) 361.

[22] Y. Lifshitz, S.R. Kasi, J.W. Rabalais, W. Eckstein, Phys. Rev. B 41 (1990) 10468.

[23] S. Sattel, J. Robertson, H. Ehrhardt, J. Appl. Phys. 82 (1997) 4566.

[24]S. Sattel, J. Gerber, and H. Ehrhardt, Phys. Stat. Sol. 154 (1996) 141.

[25] J. Robertson, J. Gerber, S. Sattel, M. Weiler, K. Jung, H. Ehrhardt, Appl. Phys. Lett. 66 (1995) 3287. 